\title{
The impact of serum 25-hydroxyvitamin D, calcium, and parathyroid hormone levels on the risk of coronary artery disease in patients with diabetes: a Mendelian randomization study
}

\author{
Songzan Chen ${ }^{1 \dagger}$, Fangkun Yang ${ }^{2 \dagger}$, Tian Xu ${ }^{1}$, Yao Wang ${ }^{1}$, Kaijie Zhang ${ }^{1}$, Guosheng Fu ${ }^{1 *}$ and Wenbin Zhang ${ }^{1 *}$ (1)
}

\begin{abstract}
Background: To investigate the causal association between serum 25-hydroxyvitamin D (25OHD), calcium (Ca), and parathyroid hormone (PTH) levels and the risk of coronary artery disease (CAD) in patients with diabetes using a Mendelian randomization approach.

Methods: Genetic signatures associated with serum 25OHD, Ca, and PTH levels were extracted from recently published genome-wide association study (GWAS), including 79,366, 39,400, 29,155 individuals, respectively. Genetic association estimates for CAD in patients with diabetes were obtained from a GWAS of 15,666 individuals with diabetes (3,968 CAD cases, 11,696 controls). The inverse-variance-weighted method was employed for the primary analysis, and other robust methods were applied for sensitivity analyses.

Results: Six, seven and five single nucleotide polymorphisms were identified as instrumental variables for serum 250HD, Ca and PTH levels, respectively. There was no significant association between genetically predicted serum $25 \mathrm{OHD}$ levels and the risk of CAD in patients with diabetes (odds ratio $(\mathrm{OR})=1.04,95 \%$ confidence interval $(\mathrm{Cl})$ : 0.58 $-1.87, P=0.888)$. Similarly, genetically predicted serum $\mathrm{Ca}(\mathrm{OR}=1.83,95 \% \mathrm{Cl}: 0.62-5.35, P=0.273)$ and PTH levels $(\mathrm{OR}=1.27,95 \% \mathrm{Cl}: 0.67-2.44, P=0.464)$ were not significantly associated with the risk of CAD in patients with diabetes. These findings were robust in sensitivity analyses.
\end{abstract}

Conclusions/interpretation: Serum 25OHD, Ca and PTH levels may not be causally associated with the risk of CAD in patients with diabetes.

Keywords: Causal association, Coronary artery disease, Diabetes, Mendelian randomization, Serum 25-hydroxyvitamin D levels, Serum calcium levels, Serum parathyroid hormone levels

*Correspondence: fugs@zju.edu.cn; 3313011@zju.edu.cn

†Songzan Chen and Fangkun Yang contributed equally to this work.

${ }^{1}$ Department of Cardiology, Key Laboratory of Cardiovascular Intervention and Regenerative Medicine of Zhejiang Province, Sir Run Run Shaw Hospital, School of Medicine, Zhejiang University, 3 East Qingchun Road, Hangzhou 310016, Zhejiang Province, China

Full list of author information is available at the end of the article

\section{Research in context}

What is already known about this subject?

- A recent Mendelian randomization study and several observational studies indicated that elevated serum calcium levels were related to increased risk of coronary artery disease in general population.

- Serum 25-hydroxyvitamin D and parathyroid hormone levels were suggested to be related to coronary original author(s) and the source, provide a link to the Creative Commons licence, and indicate if changes were made. The images or other third party material in this article are included in the article's Creative Commons licence, unless indicated otherwise in a credit line to the material. If material is not included in the article's Creative Commons licence and your intended use is not permitted by statutory regulation or exceeds the permitted use, you will need to obtain permission directly from the copyright holder. To view a copy of this licence, visit http://creativecommons.org/licenses/by/4.0/. The Creative Commons Public Domain Dedication waiver (http://creativeco mmons.org/publicdomain/zero/1.0/) applies to the data made available in this article, unless otherwise stated in a credit line to the data. 
artery disease in general population, however, Mendelian randomization studies and randomized controlled trials indicated no significant association.

\section{What is the key question?}

- Dose genetically predicted serum 25-hydroxyvitamin D, calcium and parathyroid hormone levels causally affect the risk of coronary artery disease in patients with diabetes?

\section{What are the new findings?}

- Genetically predicted serum 25-hydroxyvitamin D, calcium and parathyroid hormone levels may not be causally associated with the risk of coronary artery disease in patients with diabetes.

\section{How might this impact on clinical practice in the foreseeable future?}

- Serum 25-hydroxyvitamin D, calcium and parathyroid hormone levels may not serve as a target for prevention or treatment of coronary artery disease in patients with diabetes, though general population may benefit from calcium-lowering therapy.

\section{Background}

Coronary artery disease (CAD) and related morbidity and mortality are increasing at an alarming rate, in large part, because of increases in aging, obesity, and diabetes [1]. The clinical outcomes associated with CAD are considerably worse for patients with diabetes than for those without diabetes [2]. Therefore, The 2019 European Society of Cardiology (ESC) Guidelines have put a special emphasis on the CAD prevention in patients with diabetes [3].

Calcium (Ca) plays a critical role for almost every aspect in biological processes, such as nerve transmission, enzyme activation, hormone regulation, muscle contraction, and blood clotting [4]. Several observational studies have suggested that serum $\mathrm{Ca}$ levels are positively associated with risk of CAD $[5,6]$. Vitamin D and parathyroid hormone (PTH) are best known as master regulators of Calcium (Ca) metabolism [7]. Vitamin D motivates the absorption of dietary $\mathrm{Ca}$ and $\mathrm{PTH}$ mobilizes $\mathrm{Ca}$ from the skeleton when serum $\mathrm{Ca}$ level is low. Besides, serum 25-hydroxyvitamin D (25OHD) is an important indicator of vitamin D status [8]. Lots of evidence has been accumulated that low level of serum 25OHD levels were associated with cardiovascular risk factors, such as obesity, dyslipidemia and hypertension $[9,10]$. In addition, previous study showed that 25OHD may affect endocrine and cardiovascular systems via its nuclear receptor (25-hydroxyvitamin D receptor (VDR)) [11]. As for PTH, previous study suggested that serum PTH levels were associated with cardiovascular mortality in patients with diabetes [12]. Additionally, PTH receptors were found in the endothelial cells, the vascular smooth muscle cells and the cardiomyocytes, which indicated that PTH may serve a role in the pathophysiology of CAD [13, 14]. However, it remains unclear whether serum 25OHD, Ca and PTH levels were causally associated with CAD risk in patients with diabetes.

Mendelian randomization (MR) can avoid some of the limitations of observational studies and is not affected by disease status, thereby avoiding reverse causation bias [15]. Genetic information used to assess associations with explicit outcomes, should be free from confounding [16]. Therefore, genetic variants that influence serum 25OHD, $\mathrm{Ca}$ and PTH levels could serve as instrumental variables (IVs) to determine the impact of serum 25OHD, Ca and PTH levels on the risk of CAD in patients with diabetes. We investigated the association between serum 25OHD, $\mathrm{Ca}$ and PTH levels and the risk of CAD in patients with diabetes, which has, as far as we know, not been examined previously using the MR approach.

\section{Methods}

Study design

A two-sample MR study was designed to appraise whether the genetically determined serum $25 \mathrm{OHD}, \mathrm{Ca}$ and PTH levels were causally related to the risk of CAD in patients with diabetes. The unbiased relationship between exposures and outcomes could be estimated if the single nucleotide polymorphisms (SNPs) selected as IVs for exposures satisfied the following assumptions: (1) IVs were strongly associated with the exposures concerned; (2) IVs were independent of any possible confounders; (3) IVs must affect outcomes only via the exposures (Fig. 1) [16].

\section{Data sources}

This current two-sample MR study was based on the summary statistics from the recently published genome-wide association study (GWAS). Specifically, genetic signatures associated with serum 25OHD levels were extracted from a large GWAS in 2018, which included 79,366 individuals [17]. The genetic summary data for serum $\mathrm{Ca}$ levels were obtained from a large GWAS meta-analysis of 39,400 individuals and < 21,679 additional individuals in 2013 [18]. The genetic 


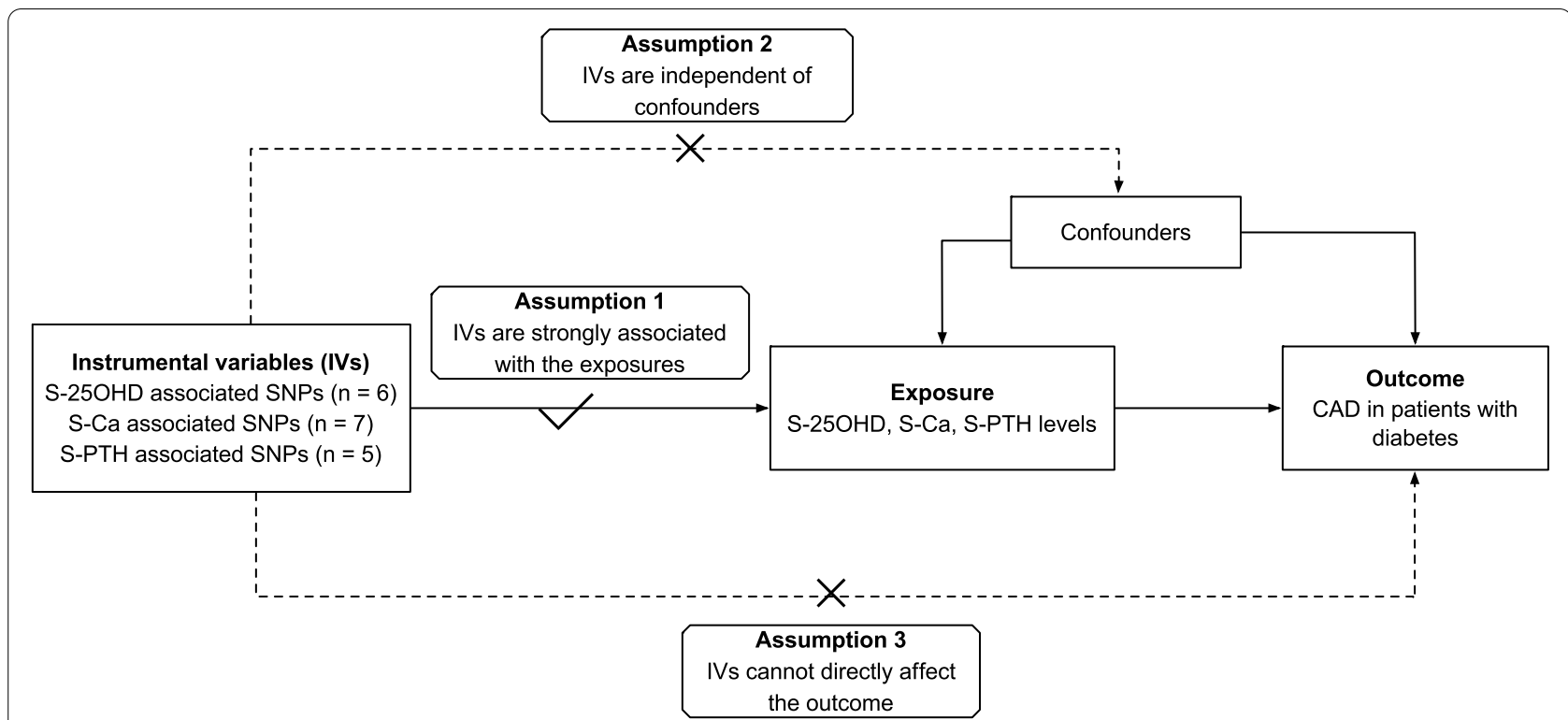

Fig. 1 Conceptual schematic of the three main assumptions that underpins the two-sample Mendelian randomization analysis of the association between serum 25-hydroxyvitamin D, calcium, parathyroid hormone levels and the risk of coronary artery disease in patients with diabetes. IV indicates instrumental variables; S-25OHD, serum 25-hydroxyvitamin D; S-Ca, serum calcium; S-PTH, serum parathyroid hormone; CAD, coronary artery disease

association with serum PTH levels were derived from a large GWAS in 2017, with up to 29,155 participants [19]. The summary statistics for CAD in patients with diabetes were obtained from a GWAS in 2018, which consisted of 15,666 individuals with diabetes $(3,968$ CAD cases, 11,696 controls) from the UK Biobank [20]. All the participants included in present study were of European ancestry.

All the studies that provided data for our analyses have received ethics approvals, and all the participants included in these studies consented to the research. This study only used publicly available data and hence no ethics approval was required.

\section{SNP selection}

To ensure a robust association between IVs and exposures, SNPs associated with serum 25OHD, Ca, PTH levels were selected from corresponding GWAS summary datasets at a genome-wide significant level $(P<$ $5 \times 10^{-8}$ ). In total, six SNPs were identified as IVs for serum 25OHD levels, seven SNPs for serum Ca levels, and five SNPs for serum PTH levels, respectively. For each trait, the selected SNPs were independent and located in different regions, which were validated by the pairwise-linkage disequilibrium test using the LDLink based on European [21]. Table 1 presented the detail information for the selected SNPs.

\section{Statistical analysis}

The conventional inverse-variance-weighted (IVW) method was employed for the major analyses. IVW method used an inverse-variance weighted formula to meta-analyze the ratio estimate of each SNPs, assuming all the SNPs were valid [22]. As a complement, weighted median, MR-Egger, and Mendelian Randomization Pleiotropy Residual Sum and Outlier (MR-PRESSO) methods for MR analyses were conducted in the sensitivity analysis. These methods may provide more robust estimates against pleiotropic instruments. For example, the weighted median method could obtain an unbiased estimate even if up to $50 \%$ of the weight came from the invalid SNPs [23]. The MR-Egger and MR-PRESSO methods could detect and correct for pleiotropy and outliers, respectively $[24,25]$.

Heterogeneity between SNPs in the IVW analysis was estimated by $\mathrm{Q}$ statistics and visually inspected by funnel plots (Figure S1-3) [23, 26]. Potential directional pleiotropy was estimated by the MR-Egger intercept test [24]. $P<0.05$ indicated the present of heterogeneity and pleiotropy, respectively. In addition, a leave-one-out analysis was performed, in which one exposure-associated SNP was removed at a time to identify any pleiotropic or outlying SNP. Furthermore, the PhenoScanner V2 was searched to detect any potential pleiotropic associations between selected SNPs and confounders [27]. The statistical power was calculated on mRnd (https://shiny.cnsge 
Table 1 Characteristics of the single-nucleotide polymorphisms associated with serum 25-hydroxyvitamin D, calcium, and parathyroid hormone levels and their associations with coronary artery disease in patients with diabetes

\begin{tabular}{|c|c|c|c|c|c|c|c|c|c|c|c|c|}
\hline \multirow[t]{2}{*}{ Traits } & \multirow[t]{2}{*}{ SNP } & \multirow[t]{2}{*}{ Chr } & \multirow[t]{2}{*}{ Nearby gene } & \multirow[t]{2}{*}{ EA } & \multirow[t]{2}{*}{$O A$} & \multirow[t]{2}{*}{ EAF } & \multicolumn{3}{|c|}{ 250HD/Calcium/PTH } & \multicolumn{3}{|c|}{$\begin{array}{l}\text { CAD in patients with } \\
\text { diabetes }\end{array}$} \\
\hline & & & & & & & Beta & SE & $P$ & Beta & SE & $P$ \\
\hline $25 \mathrm{OHD}$ & rs3755967 & 4 & GC & C & $\mathrm{T}$ & 0.72 & 0.089 & 0.002 & 4.74E-343 & 0.032 & 0.029 & 0.274 \\
\hline $25 \mathrm{OHD}$ & rs12785878 & 11 & DHCR7 & $\mathrm{T}$ & G & 0.75 & 0.036 & 0.002 & $3.80 \mathrm{E}-62$ & -0.055 & 0.033 & 0.090 \\
\hline $25 \mathrm{OHD}$ & rs10741657 & 11 & CYP2R1 & A & G & 0.40 & 0.031 & 0.002 & $2.05 \mathrm{E}-46$ & -0.001 & 0.027 & 0.972 \\
\hline $25 \mathrm{OHD}$ & rs17216707 & 20 & CYP24A1 & $\mathrm{T}$ & C & 0.79 & 0.026 & 0.003 & $8.14 \mathrm{E}-23$ & -0.033 & 0.035 & 0.344 \\
\hline $25 \mathrm{OHD}$ & rs10745742 & 12 & AMDHD1 & $\mathrm{T}$ & C & 0.40 & 0.017 & 0.002 & $1.88 \mathrm{E}-14$ & -0.019 & 0.028 & 0.495 \\
\hline $25 \mathrm{OHD}$ & rs8018720 & 14 & SEC23A & G & C & 0.18 & 0.017 & 0.003 & 4.72E-09 & 0.019 & 0.034 & 0.586 \\
\hline Calcium & rs1801725 & 3 & CASR & $\mathrm{T}$ & G & 0.15 & 0.071 & 0.004 & $8.90 \mathrm{E}-86$ & 0.052 & 0.039 & 0.186 \\
\hline Calcium & rs1570669 & 20 & CYP24A1 & G & A & 0.34 & 0.018 & 0.003 & $9.10 \mathrm{E}-12$ & -0.001 & 0.028 & 0.981 \\
\hline Calcium & rs1550532 & 2 & DGKD & $C$ & G & 0.31 & 0.018 & 0.003 & $8.20 \mathrm{E}-11$ & -0.017 & 0.028 & 0.545 \\
\hline Calcium & rs7481584 & 11 & CARS & G & A & 0.70 & 0.018 & 0.003 & $1.20 \mathrm{E}-10$ & -0.004 & 0.030 & 0.904 \\
\hline Calcium & rs780094 & 2 & GCKR & $\mathrm{T}$ & $C$ & 0.42 & 0.017 & 0.003 & $1.30 \mathrm{E}-10$ & 0.085 & 0.028 & 0.002 \\
\hline Calcium & rs7336933 & 13 & DGKH & G & A & 0.85 & 0.022 & 0.004 & $9.10 \mathrm{E}-10$ & -0.021 & 0.037 & 0.568 \\
\hline Calcium & rs10491003 & 10 & GATA3 & $\mathrm{T}$ & C & 0.09 & 0.027 & 0.005 & 4.80E-09 & -0.013 & 0.046 & 0.784 \\
\hline PTH & rs6127099 & 20 & CYP24A1 & $\mathrm{T}$ & A & 0.34 & 0.070 & 0.003 & $2.40 \mathrm{E}-72$ & 0.021 & 0.030 & 0.494 \\
\hline PTH & rs4074995 & 5 & RGS14 & G & A & 0.71 & 0.030 & 0.003 & $3.30 \mathrm{E}-23$ & 0.029 & 0.030 & 0.337 \\
\hline PTH & rs219779 & 21 & CLDN14 & G & A & 0.75 & 0.040 & 0.003 & $8.90 \mathrm{E}-22$ & -0.017 & 0.030 & 0.582 \\
\hline PTH & rs4443100 & 22 & RTDR1 & G & $C$ & 0.32 & 0.020 & 0.003 & 4.10E-11 & -0.025 & 0.029 & 0.390 \\
\hline PTH & rs73186030 & 3 & CASR & $\mathrm{T}$ & C & 0.14 & 0.030 & 0.004 & 1.20E-09 & 0.051 & 0.039 & 0.198 \\
\hline
\end{tabular}

SNP indicates single-nucleotide polymorphism, Chr chromosome, EA effect allele, OA other allele, EAF effect allele frequency, 25OHD 25-hydroxyvitamin D, PTH parathyroid hormone, $C A D$ coronary artery disease, $S E$ standard error

nomics.com/mRnd/) [28]. Based on the sample size of 15,666 and $0.46 \%$ of variance in $25 \mathrm{OHD}, 0.21 \%$ of variance in $\mathrm{Ca}$, and $0.36 \%$ of variance in $\mathrm{PTH}$ explained by the selected SNPs, our MR analyses had $80 \%$ power at an alpha rate of $5 \%$ to detect an OR of 1.88 per SD unit of 25OHD, 2.35 per SD unit of Ca, 2.01 per SD unit of PTH.

A $P$ value $<0.017$ was deemed statistically significant after the Bonferroni correction for three exposures. All the statistical analyses were performed in RStudio ( $\mathrm{R}$ version 3.6.2).

\section{Results}

Six SNPs likely to genetically determine the serum 25OHD levels were identified as IVs for serum 25OHD levels, seven SNPs for serum Ca levels, and five SNPs for serum PTH levels, respectively. Table 1 presented the genetic associations between IVs for serum 25OHD, Ca, and PTH levels and the risk of CAD in patients with diabetes. After searching in the PhenoScanner, rs780094 was found strongly associated with metabolic traits such as blood glucose, triglycerides, and total cholesterol, which may represent a pleiotropic SNP, while the remained SNPs were not related to any traditional risk factor of CAD in patients with diabetes.

\section{The effect of serum 25OHD levels on the risk of CAD in patients with diabetes}

There was no significant association between genetically predicted serum 25OHD levels, based on six SNPs, and the risk of CAD in patients with diabetes in the IVW analysis (odds ratio $(\mathrm{OR})=1.04,95 \%$ confidence interval $(\mathrm{CI}): 0.58-1.87, P=0.888)$. In line with this primary result, no significant association was observed in the sensitivity analyses using the weighted median, MR-Egger, and MR-PRESSO methods (Fig. 2). No evidence of heterogeneity was observed between the estimates of selected six SNPs $(\mathrm{Q}=5.71, P=0.336)$, and no potential directional pleiotropy was revealed in the MR-Egger intercept test $(P=0.243)$. Figure $S 4$ presented the results of leave-one-out analysis, which indicated that rs3755967 might affect the association between serum 25OHD levels and the risk of CAD in patients with diabetes (Overall $\mathrm{OR}=1.04, P=0.888$; OR after removing $\mathrm{rs} 3755967=0.47, P=0.149)$. However, rs375596 was not related to any traditional risk factor of CAD in patients with diabetes, based on the Phenoscanner search, namely, no evidence to support that rs375596 was a potential pleiotropic SNP was observed. 


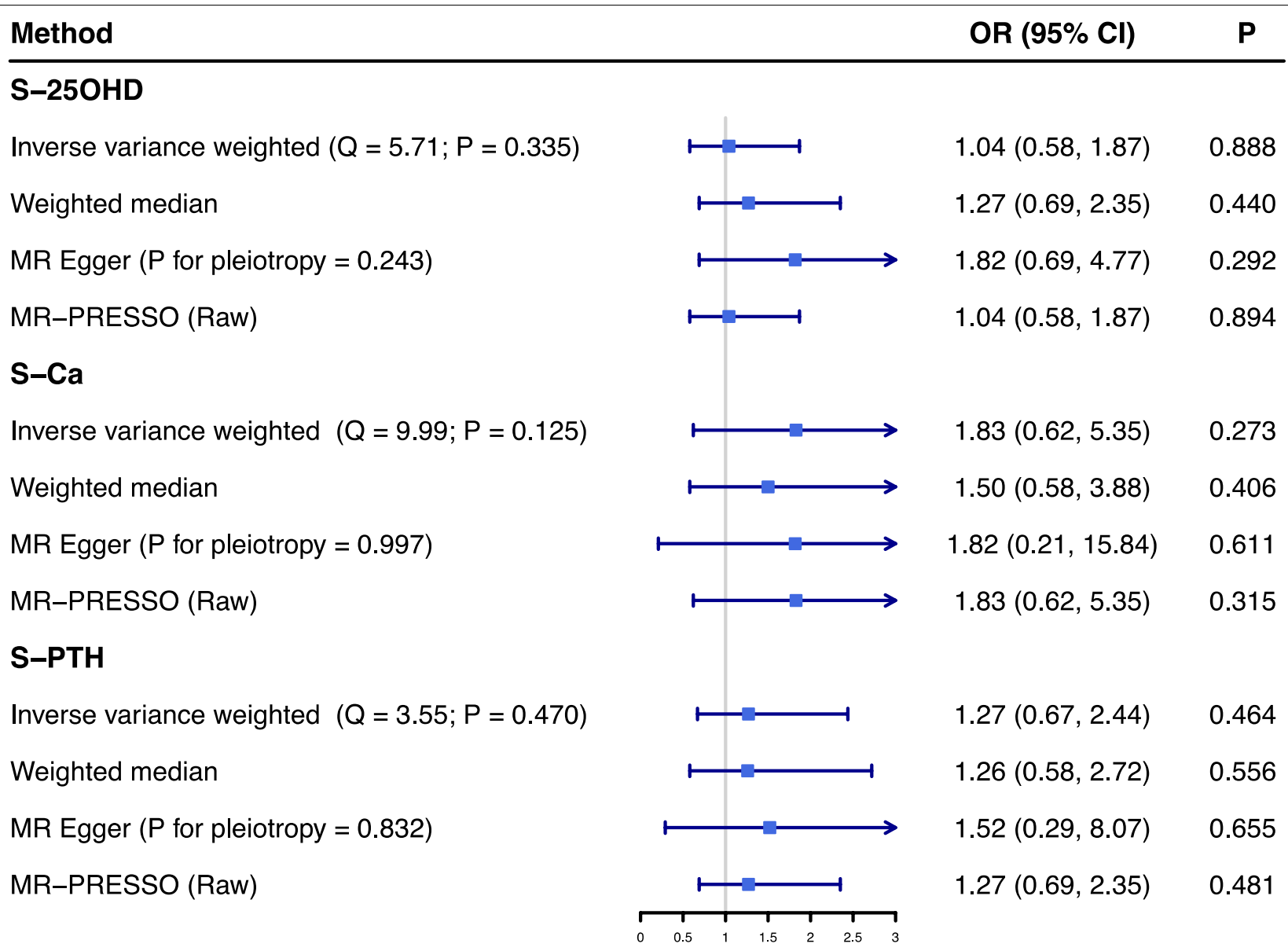

Fig. 2 Summary Mendelian randomization estimates of the associations between serum 25-hydroxyvitamin $D$, calcium, parathyroid hormone levels and the risk of coronary artery disease in patients with diabetes. OR indicates odds ratio; Cl, confidence interval; IVW, inverse variance-weighted; MR, Mendelian randomization; MR-PRESSO, MR Pleiotropy Residual Sum and Outlier; S-25OHD, serum 25-hydroxyvitamin D; S-Ca, serum calcium; S-PTH, serum parathyroid hormone

The effect of serum Ca levels on the risk of CAD in patients with diabetes

No significant association between genetic predisposition to serum Ca levels and the risk of CAD in patients with diabetes was found in the IVW analysis $(\mathrm{OR}=1.83,95 \% \mathrm{CI}: 0.62-5.35, P=0.273)$. Similar results were observed in the sensitivity analyses (Fig. 2). Q statistics suggested no evidence of heterogeneity $(Q=9.99, P=0.125)$ and the intercept of MR-Egger regression indicated no evidence of pleiotropy $(P=0.997)$. Though rs780094 was a potential pleiotropic SNP based on Phenoscanner search, the result of leave-oneout analysis indicated that association was attenuated but still insignificant (Overall OR $=1.83, P$ $=0.273$; OR after removing $\mathrm{rs} 780094=1.32, P=$ 0.529) (Figure S5).
The effect of serum PTH levels on the risk of CAD in patients with diabetes

The IVW analysis suggested no significant association between genetically determined serum PTH levels and the risk of $\mathrm{CAD}$ in patients with diabetes $(\mathrm{OR}=1.27,95 \% \mathrm{CI}$ : $0.67-2.44, P=0.464)$, which was confirmed by the sensitivity analyses using the weighted median, MR-Egger and MR-PRESSO methods (Fig. 2). In addition, no evidence of heterogeneity $(\mathrm{Q}=3.55, P=0.470)$ and pleiotropy $(P=$ 0.832 ) was observed in our study. The leave-one-out analysis indicated that this insignificant association was not disproportionally driven by any individual SNP (Figure S6).

\section{Discussion}

To the best of our knowledge, this is the first two-sample MR study to explore the association of serum 25OHD, $\mathrm{Ca}$ and PTH levels with the risk of CAD in patients with 
diabetes. The results of this MR study showed that no evidence approved causal effects of genetically determined serum 25OHD, Ca and PTH levels on CAD risk in patients with diabetes. The findings were robust in sensitivity analyses with different statistical models.

Evidence from previous multiple studies indicates that increased serum $\mathrm{Ca}$, a short term consequence of $\mathrm{Ca}$ supplementation, is associated with an increased risk and mortality of CAD [29]. Several traditional observational studies individuals with a subsequent elevation of serum Ca levels showed increased extent of coronary artery calcification [30,31], increased incidence of stroke [32], increased risk of developing heart failure [33], and an increased risk of cardiovascular mortality [34]. Possible mechanisms whereby elevated serum Ca levels may increase the risk of CAD include effects on calcification, blood coagulation, and altered gene expression induced by effects on arterial wall Ca-sensing receptor [35].

25-hydroxyvitamin $\mathrm{D}$ is the major circulating form of vitamin $\mathrm{D}$ and is used as indicator of vitamin $\mathrm{D}$ status [36]. As critical regulators for Ca metabolism, 25OHD can exert a biological effect mainly through regulating intracellular and extracellular $\mathrm{Ca}$ concentrations [7]. In addition to the regulation of $\mathrm{Ca}$ metabolism, 25OHD is involved in cell proliferation and differentiation and exerts an immunomodulatory and anti-inflammatory property. Evidence from previous studies revealed that 25OHD deficiency resulted in the increase expression of inflammatory factors, such as interleukin 6, interleukin 8, and C-reactive protein [37], which play important roles in CAD. Furthermore, low serum 25OHD levels are associated with obesity, dyslipidemia, the metabolic syndrome, and hypertension, which are all well-established CAD risk factors $[9,10]$. It was shown that lower serum bioavailable and free 25OHD levels were associated with prevalent cardiovascular mortality independent of other recognized cardiovascular risk factors [38]. Vitamin D deficiency might be considered a so-called "nonclassical" CAD risk factor in studies without a specific focus on diabetes [39, 40]. However, the two recent large scale RCTs failed to demonstrate a beneficial effect of 25OHD on CAD [41, 42].

Consistently, PTH is a key regulator of Ca homeostasis. With further in-depth studies, PTH appears to play a role in the cardiovascular system [13]. A number of epidemiologic studies showed associations of mild hyperparathyroidism with hypertension, endothelial dysfunction and metabolic syndrome, which were associated to an increased incidence of CAD [13]. A recent observational cohort study showed a significant association between PTH levels and cardiovascular mortality in patients with diabetes, which was true after adjustment for classical
CAD risk factors [12]. However, a systematic review and meta-analysis of prospective studies suggested no significant association between serum PTH levels and CAD [43].

The association of serum 25OHD, Ca and PTH levels with the risk of CAD is a controversial issue, and the findings seem to vary depending on the investigated population. Our study focuses on a population with diabetes. Until then, very little research has been done in this area. We subsequently performed a MR study to explore the association of serum 25OHD, $\mathrm{Ca}$ and PTH levels with the risk of CAD in patients with diabetes. To date, randomized controlled trials (RCTs) remain the gold standard to establish causal relationships [44]. However, RCTs cannot always be conducted, because they can be excessively costly and require a long period to demonstrate the effect on CAD events [15]. When the number of confounders is too large, or when confounders are uncertain, the observed associations may not reflect the causality but may arise as the result of confounding or reverse causation $[45,46]$. In the absence of high-quality RCTs and given the inconclusive results from the existing observational studies, MR may serve as a time- and cost-efficient alternative approach and contribute to its increasing popularity for assessing and screening for potentially causal associations [15, 47]. A recent study without a specific focus on diabetes has used this technique to demonstrate that a genetic predisposition to higher serum $\mathrm{Ca}$ levels was associated with increased risk of CAD and myocardial infarction [48]. In a large, well-powered MR study, genetically lowered serum 25OHD levels were not associated with increased risk of CAD [49]. Melhus et al. used the MR approach to demonstrate that genetic predisposition to higher serum PTH concentrations did not appear to be an independent risk factor for CAD among the general population [50].

Consistent with the results in the general population $[49,50]$, we found no evidence to support the causal association of serum 25OHD and PTH levels with the risk of CAD in patients with diabetes with the aid of the hitherto largest GWAS meta-analysis. Unlike previous studies [48, 51], our results provided evidence against a causal role for elevated serum Ca levels in CAD susceptibility, because of our focus on patients with diabetes. Serum 25OHD, Ca, and PTH levels should therefore not be regarded as independent risk factors for CAD in patients with diabetes. Those associations reported in the past might be due to indirect effects by residual confounding. Serum 25OHD, Ca and PTH levels are more likely biomarkers rather than causal risk factors for CAD in patients with diabetes. 
The major strength of this study is the MR design, using 25OHD-, Ca-, and PTH-related SNPs and SNPs-CAD in patients with diabetes from a recently published large-scale GWAS, which minimized reverse causation bias and confounding that can produce false associations in traditional observational studies. Using data from a large genetic consortium for 25OHD $(n=79,366)$, serum Ca $(n=39,400)$, PTH $(n=29,155)$ levels and CAD risk $(3,968$ cases and 11,696 controls) in patients with diabetes has enabled us to more precisely test our study hypothesis than if we had used individual-level data from a small study. Furthermore, in the absence of large-scale, longterm RCT data, our findings provide strong evidence against a causal role for low serum 25OHD levels, elevated serum $\mathrm{Ca}$ and PTH levels in CAD susceptibility in patients with diabetes.

Our study also has several potential limitations that deserve discussion. First, potential pleiotropy is difficult to exclude in any MR study. However, all the results analyzed with other more robust methods in the sensitivity analyses were consistent with the primary results. The null result could also be explained by canalization, which is defined as compensatory feedback interactions [52]. Second, we only investigated explore the association of serum 25OHD, Ca and PTH levels with the risk of CAD in patients with diabetes from a genetic point of view. We were unable to examine whether there are any interactions between genetically predicted serum 25OHD, Ca and PTH levels and lifestyle/environmental factors on the risk of CAD in patients with diabetes. Third, the examined GWAS were primarily conducted in individuals of European ancestry, which confines the transferability of the present findings to other ethnicities. Thus, studies in a causal association are warranted to verify the present conclusions among individuals of different ancestries. Finally, our results did not reach a statistical power of $80 \%$, which probably caused by the low variance in exposures explained by the selected SNPs and the insufficient sample size. Therefore, it should be cautious to make the conclusion and we had to admit that there was a possibility that false-negative results may occur.

\section{Conclusions}

Overall, serum 25OHD, Ca, and PTH levels may not be causally associated with the risk of CAD in patients with diabetes. Serum 25OHD, Ca and PTH levels may not serve as a target for prevention or treatment of CAD in patients with diabetes. Further investigations are warranted to validate our findings.

\section{Availability of supporting data}

The datasets analyzed in this study are publicly available summary statistics.

\begin{abstract}
Abbreviations
Ca: Calcium; CAD: Coronary artery disease; ESC: European Society of Cardiology; GWAS: Genome-wide association study; IV: Instrumental variables; IVW: Inverse-variance-weighted; MR: Mendelian randomization; MRPRESSO: Mendelian Randomization Pleiotropy Residual Sum and Outlier; PTH: Parathyroid hormone; VDR: 25-hydroxyvitamin D receptor; 25OHD: 25-hydroxyvitamin D.
\end{abstract}

\section{Supplementary Information}

The online version contains supplementary material available at https://doi. org/10.1186/s12937-021-00735-z.

Additional file 1: Figure S1. Funnel plot of the Mendelian randomization estimate for the association between serum 25-hydroxyvitamin D levels and the risk of CAD in patients with diabetes. Figure S2. Funnel plot of the Mendelian randomization estimate for the association between serum calcium levels and the risk of CAD in patients with diabetes. Figure S3. Funnel plot of the Mendelian randomization estimate for the association between serum parathyroid hormone levels and the risk of CAD in patients with diabetes. Figure S4. Leave-one-out analysis of the association between serum 25-hydroxyvitamin D levels and the risk of coronary artery disease in patients with diabetes. Figure S5. Leave-one-out analysis of the association between serum calcium levels and the risk of coronary artery disease in patients with diabetes. Figure S6. Leave-one-out analysis of the association between serum parathyroid hormone levels and the risk of coronary artery disease in patients with diabetes.

\section{Acknowledgements}

Not applicable.

\section{Authors' contributions}

Songzan Chen, Guosheng Fu and Wenbin Zhang designed the study, and wrote the analysis plan. Songzan Chen and Fangkun Yang undertook analyses and all authors interpreted the results in the study. Songzan Chen and Tian Xu wrote the first draft of the manuscript with critical revisions from Fangkun Yang, Yao Wang and Kaijie Zhang. All authors gave final approval of the version to be published.

Funding

This study was supported by: National Natural Science Foundation of China (82070408).

\section{Declarations}

Ethics approval and consent to participate

Ethical approval and Consent to participate are not needed for this current study because it is a secondary analysis of previously published data.

Consent for publication

Not applicable.

Competing interests

The authors declare no competing interests.

Author details

${ }^{1}$ Department of Cardiology, Key Laboratory of Cardiovascular Intervention and Regenerative Medicine of Zhejiang Province, Sir Run Run Shaw Hospital, School of Medicine, Zhejiang University, 3 East Qingchun Road, Hangzhou 310016, Zhejiang Province, China. ${ }^{2}$ Department of Cardiology, Ningbo First Hospital, School of Medicine, Zhejiang University, Ningbo 315010, China. 
Received: 13 November 2020 Accepted: 26 August 2021

Published online: 03 October 2021

\section{References}

1. Moran $\mathrm{AE}$, Forouzanfar MH, Roth $\mathrm{GA}$, et al. Temporal trends in ischemic heart disease mortality in 21 world regions, 1980 to 2010: The Global Burden of Disease 2010 study. Circulation. 2014;129(14):1483-92. https:// doi.org/10.1161/CIRCULATIONAHA. 113.004042.

2. Rawshani A, Rawshani A, Franzen S, et al. Risk factors, mortality, and cardiovascular outcomes in patients with type 2 diabetes. N Engl J Med. 2018:379(7):633-44. https://doi.org/10.1056/NEJMoa1800256.

3. Cosentino F, Grant PJ, Aboyans V, et al. 2019 ESC Guidelines on diabetes, pre-diabetes, and cardiovascular diseases developed in collaboration with the EASD. Eur Heart J. 2020;41 (2):255-323. https://doi.org/10.1093/ eurhearti/ehz486.

4. Baird GS. Ionized calcium. Clin Chim Acta. 2011;412(9-10):696-701. https://doi.org/10.1016/j.cca.2011.01.004.

5. Reid IR, Gamble GD, Bolland MJ. Circulating calcium concentrations, vascular disease and mortality: A systematic review. J Intern Med. 2016;279(6):524-40. https://doi.org/10.1111/joim.12464.

6. Rohrmann S, Garmo H, Malmstrom H, et al. Association between serum calcium concentration and risk of incident and fatal cardiovascular disease in the prospective AMORIS study. Atherosclerosis. 2016;251:85-93. https://doi.org/10.1016/j.atherosclerosis.2016.06.004.

7. Wenclewska S, Szymczak-Pajor I, Drzewoski J, Bunk M, Sliwinska A. Vitamin D supplementation reduces both oxidative DNA damage and insulin resistance in the elderly with metabolic disorders. Int J Mol Sci. 2019;20(12):2891. https://doi.org/10.3390/ijms20122891.

8. Herrmann M, Farrell CL, Pusceddu I, Fabregat-Cabello N, Cavalier E. Assessment of vitamin D status - a changing landscape. Clin Chem Lab Med. 2017;55(1):3-26. https://doi.org/10.1515/cclm-2016-0264.

9. Bahrami LS, Ranjbar G, Norouzy A, Arabi SM. Vitamin D supplementation effects on the clinical outcomes of patients with coronary artery disease: A systematic review and meta-analysis. Sci Rep. 2020;10(1):12923. https:// doi.org/10.1038/s41598-020-69762-w.

10. Grandi NC, Breitling LP, Brenner H. Vitamin D and cardiovascular disease: Systematic review and meta-analysis of prospective studies. Prev Med. 2010;51(3-4):228-33. https://doi.org/10.1016/j.ypmed.2010.06.013.

11. Ramagopalan SV, Heger A, Berlanga AJ, et al. A ChIP-seq defined genome-wide map of vitamin $D$ receptor binding: Associations with disease and evolution. Genome Res. 2010;20(10):1352-60. https://doi. org/10.1101/gr.107920.110

12. Brandtner EM, Muendlein A, Leiherer A, et al. (2020) Serum parathyroid hormone predicts mortality in coronary angiography patients with type 2 diabetes. J Clin Endocrinol Metab 105(11):e3874-81. https://doi.org/10. 1210/clinem/dgaa512

13. Pepe J, Cipriani C, Sonato C, Raimo O, Biamonte F, Minisola S. Cardiovascular manifestations of primary hyperparathyroidism: A narrative review. Eur J Endocrinol. 2017;177(6):R297-308. https://doi.org/10.1530/ EJE-17-0485.

14. Fitzpatrick LA, Bilezikian JP, Silverberg SJ. Parathyroid hormone and the cardiovascular system. Curr Osteoporos Rep. 2008;6(2):77-83. https://doi. org/10.1007/s11914-008-0014-8.

15. Sekula P, Del Greco MF, Pattaro C, Kottgen A. Mendelian randomization as an approach to assess causality using observational data. J Am Soc Nephrol. 2016;27(11):3253-65. https://doi.org/10.1681/ASN.2016010098.

16. Holmes MV, Ala-Korpela M, Smith GD. Mendelian randomization in cardiometabolic disease: Challenges in evaluating causality. Nat Rev Cardiol. 2017;14(10):577-90. https://doi.org/10.1038/nrcardio.2017.78.

17. Jiang $X$, O'Reilly PF, Aschard $H$, et al. Genome-wide association study in 79,366 European-ancestry individuals informs the genetic architecture of 25-hydroxyvitamin D levels. Nat Commun. 2018;9(1):260. https://doi.org/ 10.1038/s41467-017-02662-2.

18. O'Seaghdha $\mathrm{CM}$, Wu H, Yang $\mathrm{Q}$, et al. Meta-analysis of genome-wide association studies identifies six new Loci for serum calcium concentrations. PLoS Genet. 2013:9(9): e1003796. https://doi.org/10.1371/journal.pgen.1003796.

19. Robinson-Cohen C, Lutsey PL, Kleber ME, et al. Genetic variants associated with circulating parathyroid hormone. J Am Soc Nephrol. 2017;28(5):1553-65. https://doi.org/10.1681/ASN.2016010069.
20. Fall T, Gustafsson S, Orho-Melander M, Ingelsson E. Genome-wide association study of coronary artery disease among individuals with diabetes: The UK Biobank. Diabetologia. 2018;61(10):2174-9. https://doi.org/10. 1007/s00125-018-4686-z.

21. Myers TA, Chanock SJ, Machiela MJ. LDlinkR: An R package for rapidly calculating linkage disequilibrium statistics in diverse populations. Front Genet. 2020;11:157. https://doi.org/10.3389/fgene.2020.00157.

22. Burgess S, Butterworth A, Thompson SG. Mendelian randomization analysis with multiple genetic variants using summarized data. Genet Epidemiol. 2013;37(7):658-65. https://doi.org/10.1002/gepi.21758.

23. Bowden J, Davey Smith G, Haycock PC, Burgess S. Consistent estimation in Mendelian randomization with some invalid instruments using a weighted median estimator. Genet Epidemiol. 2016;40(4):304-14. https:// doi.org/10.1002/gepi.21965.

24. Bowden J, Davey Smith G, Burgess S. Mendelian randomization with invalid instruments: Effect estimation and bias detection through Egger regression. Int J Epidemiol. 2015;44(2):512-25. https://doi.org/10.1093/ ije/dyv080.

25. Verbanck M, Chen CY, Neale B, Do R. Detection of widespread horizontal pleiotropy in causal relationships inferred from Mendelian randomization between complex traits and diseases. Nat Genet. 2018;50(5):693-8. https://doi.org/10.1038/s41588-018-0099-7.

26. Sterne JA, Sutton AJ, loannidis JP, et al. Recommendations for examining and interpreting funnel plot asymmetry in meta-analyses of randomised controlled trials. BMJ. 2011;343: d4002. https://doi.org/10.1136/bmj.d4002.

27. Staley JR, Blackshaw J, Kamat MA, et al. PhenoScanner: A database of human genotype-phenotype associations. Bioinformatics. 2016;32(20):3207-9. https://doi.org/10.1093/bioinformatics/ btw373.

28. Burgess S. Sample size and power calculations in Mendelian randomization with a single instrumental variable and a binary outcome. Int J Epidemiol. 2014;43(3):922-9. https://doi.org/10.1093/ije/dyu005.

29. Reid IR, Birstow SM, Bolland MJ. Calcium and cardiovascular disease. Endocrinol Metab. 2017;32(3):339-49. https://doi.org/10.3803/EnM.2017. 32.3.339.

30. Kwak SM, Kim JS, Choi Y, et al. Dietary intake of calcium and phosphorus and serum concentration in relation to the risk of coronary artery calcification in asymptomatic adults. Arterioscler Thromb Vasc Biol. 2014;34(8):1763-9. https://doi.org/10.1161/ATVBAHA.114.303440.

31. Anderson JJ, Kruszka B, Delaney JA, et al. Calcium intake from diet and supplements and the risk of coronary artery calcification and its progression among older adults: 10-year follow-up of the multi-ethnic study of atherosclerosis (MESA). J Am Heart Assoc. 2016;5(10):e003815-27. https:// doi.org/10.1161/JAHA. 116.003815

32. Mao PJ, Zhang C, Tang L, et al. Effect of calcium or vitamin D supplementation on vascular outcomes: A meta-analysis of randomized controlled trials. Int J Cardiol. 2013;169(2):106-11. https://doi.org/10.1016/j.ijcard. 2013.08.055.

33. Lutsey PL, Alonso A, Michos ED, et al. Serum magnesium, phosphorus, and calcium are associated with risk of incident heart failure: The Atherosclerosis Risk in Communities (ARIC) Study. Am J Clin Nutr. 2014;100(3):756-64. https://doi.org/10.3945/ajcn.114.085167.

34. Shiyovich A, Plakht Y, Gilutz H. Serum calcium levels independently predict in-hospital mortality in patients with acute myocardial infarction. Nutr Metab Cardiovasc Dis. 2018;28(5):510-6. https://doi.org/10.1016/j. numecd.2018.01.013.

35. Reid IR, Bristow SM, Bolland MJ. Cardiovascular complications of calcium supplements. J Cell Biochem. 2015;1 16(4):494-501. https://doi.org/10. 1002/jcb.25028.

36. Zerwekh JE. Blood biomarkers of vitamin D status. Am J Clin Nutr. 2008;87(4):1087S-1091S. https://doi.org/10.1093/ajcn/87.4.1087S.

37. Esteghamati A, Aryan Z, Esteghamati A, Nakhjavani M. Vitamin D deficiency is associated with insulin resistance in nondiabetics and reduced insulin production in type 2 diabetics. Horm Metab Res. 2015;47(4):273-9. https://doi.org/10.1055/s-0034-1389903.

38. Yu C, Xue H, Wang L, et al. Serum bioavailable and free 25-hydroxyvitamin D levels, but not its total level, are associated with the risk of mortality in patients with coronary artery disease. Circ Res. 2018;123(8):996-1007. https://doi.org/10.1161/CIRCRESAHA.118.313558.

39. Iyngkaran $\mathrm{P}$, Chan W, Liew D, et al. Risk stratification for coronary artery disease in multi-ethnic populations: Are there broader considerations 
for cost efficiency? World J Methodol. 2019;9(1):1-19. https://doi.org/10. 5662/wjm.v9.i1.1.

40. Kunadian V, Ford GA, Bawamia B, Qiu W, Manson JE. Vitamin D deficiency and coronary artery disease: A review of the evidence. Am Heart J. 2014;167(3):283-91. https://doi.org/10.1016/j.ahj.2013.11.012.

41. Scragg R, Stewart AW, Waayer D, et al. Effect of monthly high-dose vitamin $\mathrm{D}$ supplementation on cardiovascular disease in the vitamin D assessment study: A randomized clinical trial. JAMA Cardiol. 2017;2(6):608-16. https://doi.org/10.1001/jamacardio.2017.0175.

42. Manson JE, Bassuk SS, Lee IM, et al. The VITamin D and OmegA-3 TriaL (VITAL): Rationale and design of a large randomized controlled trial of vitamin $\mathrm{D}$ and marine omega-3 fatty acid supplements for the primary prevention of cancer and cardiovascular disease. Contemp Clin Trials. 2012;33(1):159-71. https://doi.org/10.1016/j.cct.2011.09.009.

43 van Ballegooijen AJ, Reinders I, Visser M, Brouwer IA. Parathyroid hormone and cardiovascular disease events: A systematic review and meta-analysis of prospective studies. Am Heart J. 2013;165(5):655-64. https://doi.org/ 10.1016/j.ahj.2013.02.014. 664 e651-655.

44. Rivara MB, Mehrotra R. Timing of dialysis initiation: What has changed since IDEAL? Semin Nephrol. 2017;37(2):181-93. https://doi.org/10.1016/j. semnephrol.2016.12.008.

45. Evans DM, Davey Smith G. Mendelian randomization: New applications in the coming age of hypothesis-free causality. Annu Rev Genomics Hum Genet. 2015;16:327-50. https://doi.org/10.1146/annur ev-genom-090314-050016.

46. Jansen H, Samani NJ, Schunkert H. Mendelian randomization studies in coronary artery disease. Eur Heart J. 2014;35(29):1917-24. https://doi.org/ 10.1093/eurheartj/ehu208.
47. Kawashiri MA, Tada H, Nomura A, Yamagishi M. Mendelian randomization: Its impact on cardiovascular disease. J Cardiol. 2018;72(4):307-13. https:// doi.org/10.1016/j.jjcc.2018.04.007.

48. Larsson SC, Burgess S, Michaelsson K. Association of genetic variants related to serum calcium levels with coronary artery disease and myocardial infarction. JAMA. 2017;318(4):371-80. https://doi.org/10.1001/jama. 2017.8981.

49. Manousaki D, Mokry LE, Ross S, Goltzman D, Richards JB. Mendelian randomization studies do not support a role for vitamin $\mathrm{D}$ in coronary artery disease. Circ Cardiovasc Genet. 2016;9(4):349-56. https://doi.org/10.1161/ CIRCGENETICS.116.001396.

50. Melhus H, Michaelsson K, Larsson SC. Serum parathyroid hormone and risk of coronary artery disease: Exploring causality using Mendelian randomization. J Clin Endocrinol Metab. 2019;104(11):5595-600. https:// doi.org/10.1210/jc.2019-01063.

51. Xu L, Lin SL, Schooling CM. A Mendelian randomization study of the effect of calcium on coronary artery disease, myocardial infarction and their risk factors. Sci Rep. 2017;7:42691. https://doi.org/10.1038/srep4 2691.

52. Mokry LE, Ahmad O, Forgetta V, Thanassoulis G, Richards JB. Mendelian randomisation applied to drug development in cardiovascular disease: A review. J Med Genet. 2015;52(2):71-9. https://doi.org/10.1136/jmedg enet-2014-102438.

\section{Publisher's Note}

Springer Nature remains neutral with regard to jurisdictional claims in published maps and institutional affiliations.
Ready to submit your research? Choose BMC and benefit from:

- fast, convenient online submission

- thorough peer review by experienced researchers in your field

- rapid publication on acceptance

- support for research data, including large and complex data types

- gold Open Access which fosters wider collaboration and increased citations

- maximum visibility for your research: over 100M website views per year

At BMC, research is always in progress.

Learn more biomedcentral.com/submissions 